\title{
Association of daytime napping in relation to risk of diabetes: evidence from a prospective study in Zhejiang, China
}

Hao Wang ${ }^{1}$, Lingli Chen², Dun Shen², Yuan Cao², Xiaoyi Zhang ${ }^{2}$, Kaixu Xie ${ }^{2}$, Chunmei Wang ${ }^{2}$, Shuiqing Zhu ${ }^{2}$, Yu Guo ${ }^{3}$, Bragg Fiona ${ }^{4}, \mathrm{Min}_{\mathrm{Yu}}{ }^{1 *}$, Zhengming Chen ${ }^{4}$ and Liming Li ${ }^{5}$

\begin{abstract}
Background: Diabetes was a major risk factor for numerous chronic diseases. However, the associations between daytime napping and diabetes in the existing literature is still inconsistent.

Methods: The analysis included 53,916 participants aged 30-79 years of the China Kadoorie Biobank prospective study from Tongxiang. Incident diabetes were identified through linkage with incident diabetes surveillance systems, health insurance system, and death registries. Cox regressions were used to estimate the associations of daytime napping with diabetes.

Results: $5.11 \%$ of participants reported habitual daytime napping. During 488,233 person-years (median 9.4 years) of follow-up, 3333 incident diabetes, including 1249 males and 2084 females, were documented. After adjusting for socio-demographic status, behavioral lifestyle, BMI, waist circumference and snoring, as comparison with those without daytime napping, the hazard ratios for risk of diabetes were 1.39 ( $95 \% \mathrm{Cl} 1.21-1.59)$. The corresponding figures for males and females were $1.45(95 \% \mathrm{Cl} 1.20-1.74)$ and $1.30(95 \% \mathrm{Cl} 1.05-1.59)$, respectively. The corresponding figures for postmenopausal and premenopausal females were $1.41(95 \% \mathrm{Cl} 1.08-1.80)$ and $1.13(95 \% \mathrm{Cl} 0.78-1.59)$, respectively.
\end{abstract}

Conclusions: Habitual daytime napping is positively associated with risk of diabetes in adults, except premenopausal females.

Keywords: Daytime napping, Diabetes, Prospective study, China

\section{Background}

Diabetes was a major risk factor for numerous chronic diseases, including heart disease, ischemic stroke and renal disease $[1,2]$. Nearly 463 million adults aged 20-79 years had diabetes worldwide, of whom three quarters lived in low and middle income countries [3]. China had the highest number of diabetics, which will

\footnotetext{
*Correspondence: myu@cdc.zj.cn

1 Department of NCDs Control and Prevention, Zhejiang Provincial Center

for Diseases Control and Prevention, \#3399 Binsheng Road, Binjiang

District, Hangzhou, Zhejiang Province, China

Full list of author information is available at the end of the article
}

continue to increase $[3,4]$. A nationally representative survey indicated that the prevalence of diabetes was $10.9 \%$ among adults over 18 years in China [5]. The estimated cost of diabetes in 2015 was 1.3 trillion dollars, accounting for approximately $1.8 \%$ of global gross domestic product [6].

Daytime napping was defined as a short sleep typically taken in the early afternoon [7]. It was one of the common behavioral habits worldwide, especially among Chinese adults. In China, napping is thought as part of healthy lifestyle and has been deeply embedded into the culture [8]. The benefit of napping included sleepiness reduction, memory consolidation, cognitive performance 
enhancement, boost in emotional stability, and endurance performance improvement [9]. 20.3\% of 30-79 years old adults are reported to be habitual napper in China [10]. However, the association of daytime napping with diabetes from published studies remain inconsistent. While one prospective cohort study suggested that daytime napping was associated with an increased diabetes risk in British population [11], another prospective study indicated that null significant association was found in Finnish population [12]. Hence, the purpose of this study was to examine the association of daytime napping with risk of incident diabetes using data from Tongxiang Zhejiang in the China Kadoorie Biobank.

\section{Methods}

\section{Study population and design}

CKB study is a nationwide, prospective cohort study. Detailed information on the CKB study design, method, and participants have been reported previously [13-15]. The data utilized in the present study came from Tongxiang, one of the 10 regions in China. Briefly, 57704 participants aged 30-79 years old were recruited and participated in the baseline survey between August 2004 and January 2008.

\section{Assessment of daytime napping}

Daytime napping was assessed through the question "Do you usually take a daytime nap?" Answer options included "Yes usually", "Yes, but only in summer", and "None". Participants who chose the first option were defined as habitual daytime nappers. Participants who chose the third option were defined as non-nappers.

\section{Assessment of covariates}

Detailed covariates information was collected at baseline by means of a standardized questionnaire, including sociodemographic characteristics (age, sex, education level, marital status, and household income), lifestyle behaviors (cigarette consumption, alcohol consumption, physical activity, intakes of fresh fruit, vegetable and meat, sleep duration and snoring), personal medical history (cancer, stroke, diabetes, and heart attack), family history of diabetes, and women menopause status. Height, weight, and waist circumference (WC) were measured using calibrated instruments by trained staff.

Standing height was measured to the nearest $0.1 \mathrm{~cm}$ with a stadiometer. Weight was measured to the nearest $0.1 \mathrm{~kg}$ with a body composition analyzer (TANITA-TBF300GS), subtracting the weight of clothing according to season (0.5 kg in summer and 2.0-2.5 kg in winter). Body mass index (BMI) was calculated as weight in kilograms divided by the square of height in meters, and obesity was defined as $\mathrm{BMI} \geq 25.0 \mathrm{~kg} / \mathrm{m}^{2}$ [16]. WC was measured to the nearest $0.1 \mathrm{~cm}$ with a non-stretchable tape measure. WC was measured at the midpoint between the lowest rib margin and the iliac crest. A non-fasting venous blood sample was collected. Immediate on-site testing of plasma glucose level was undertaken. Random blood glucose levels were measured using the SureStep Plus System (Johnson \& Johnson, New Brunswick, NJ, USA) Participants who did not report a history of diabetes but who had random blood glucose $\geq 11.1 \mathrm{mmol} / \mathrm{l}$ or fasting blood glucose $\geq 7.0 \mathrm{mmol} / \mathrm{l}$ were defined as having screen-detected diabetes.

In the present study, participants with self-reported history of physician-diagnosed chronic diseases (163 with cancers, 349 with strokes, 464 with heart diseases and 1380 with diabetes) and 1432 participants with screendetected diabetes at baseline, were excluded, yielding a total of 53916 (22,573 men, 31,343 women) participants in the final analysis.

\section{Follow-up for incident diabetes}

All the participants were followed up from the date of baseline survey until the date of diabetes events, death, or 31 December 2015. Incident diabetes were obtained periodically through linkage with local diabetes surveillance system, death registries, and with national insurance electronic system, covering more than $98 \%$ study participants in Tongxiang. Trained staff, blinded to the baseline information, coded all cases with the 10th revision of International Classification of Diseases (ICD-10). For the present analysis, we included diabetes cases as E10-E14.

\section{Statistical analysis}

Percentage and mean values of characteristics at baseline were calculated according to answer of daytime napping. Cox proportional hazards regression was used to estimate adjusted hazard ratios (HRs) of diabetes in relation to daytime napping and $95 \%$ confidence intervals. Four different models were performed to calculate HRs of diabetes risk. Models were stratified by age at baseline (in 5-year intervals), and sex. In model 1, HRs were adjusted for age and sex. In model 2, HRs was further adjusted for education level (no formal education, primary school, middle school, and high school or above), household income ( $<19,999$ yuan, 20,000-34,999 yuan, $\geq 35,000$ yuan), marital status, cigarettes consumption (never, occasional, former, and current regular), alcohol consumption (never, occasional, former, and current regular), meat consumption (daily and non-daily), fresh fruits consumption (daily and non-daily), fresh vegetables consumption(daily and non-daily), physical activity (metabolic equivalent of tasks hours/day; continuous), and sleep duration (continuous). In model 3, HRs were further adjusted for BMI (continuous) and WC 
(continuous). In model 4, HRs were further adjusted for self-reported snoring (none, occasional, and habitual). In subgroup analyses, participants were divided into nonobese group and obese group according to their BMI, and females were divided into premenopausal group and post-menopausal group according to their menopause status. In sensitivity analyses, the first 2 years of followup was excluded to reduce the effect of reverse causality, and those with diabetes family history was excluded to reduce the effect of family history. All statistical analysis was calculated by using SAS version 9.3, and a two-sided $P<0.05$ was defined as statistically significant.

\section{Results}

\section{Characteristics of participants}

Of the 53916 participants included, the mean (SD) baseline age was $52.0(9.9)$ years and $58.1 \%$ was female. $37.8 \%$ of participants' household income were more than 35,000 (yuan). 28.0\% of participants were current regular smokers. $17.1 \%$ of participants were current regular drinkers. The average of total physical activity of participants was $30.6 \mathrm{MET}-\mathrm{h} / \mathrm{d}$. $15.2 \%$ of participants consumed meat daily. $6.7 \%$ of participants consumed fresh fruits daily. 93.8\% of participants consumed vegetables daily. The average of BMI was $22.9 \mathrm{~kg} / \mathrm{m}^{2}$ and the average of WC was $76.5 \mathrm{~cm}$. The average of sleep duration of participants was $7.6 \mathrm{~h}$ daily. $53.7 \%$ of women was postmenopausal. As compared with non-nappers, habitual nappers were more likely to be older, to be males, to be well-educated, to have more household income, to be current regular drinker, to be physically inactive, to consume meat and fruit frequently, to have a higher BMI and WC, and to have more sleep duration, and less likely to be current regular smokers. No significant difference of the proportion of postmenopausal females between three groups. $(P=0.86)$. (Table 1$)$.

\section{Percentage of daytime napping}

Of all participants, the proportion of habitual daytime napping, napping only in summer, and non-napping were $5.11 \%, 32.72 \%$ and $62.15 \%$, respectively. The percentage of habitual daytime napping among 30-49 years, 50-59 years, and 60-79 years group were 4.49 (95\% CI 4.22-4.75), 4.63 (95\% CI 4.33-4.93), and 7.02 (95\% CI 6.57-7.48), respectively. The percentage of habitual daytime napping increased with an increase of age $(P<0.0001)$. The percentage of habitual daytime napping was higher among males than females $(7.17 \%$ vs. $3.63 \%)$ $(P<0.0001)$. (Table 2$)$.

\section{Association of daytime napping with diabetes}

During 488,233 person-years (median 9.4 years) of follow-up, 3333 incident diabetes, 1249 were males, and 2084 were females were documented. For habitual daytime napping, after adjusting for socio-demographic status, cigarettes, alcohol, physical activity, dietary consumption and sleep duration, in comparison with

Table 1 Baseline characteristics of the participants by frequency of daytime napping

\begin{tabular}{|c|c|c|c|c|c|}
\hline \multirow[t]{2}{*}{ Characteristics } & \multirow[t]{2}{*}{ Overall $(n=53916)$} & \multicolumn{3}{|l|}{ Daytime napping } & \multirow[t]{2}{*}{$P$} \\
\hline & & Habitual $(n=2757)$ & $\begin{array}{l}\text { Only in summer } \\
(n=17,648)\end{array}$ & $\begin{array}{l}\text { Non-napping } \\
(n=33,511)\end{array}$ & \\
\hline Mean age (years) & $52.0 \pm 9.9$ & $53.1 \pm 10.8$ & $52.9 \pm 9.8$ & $51.3 \pm 9.7$ & $<0.0001$ \\
\hline Females (\%) & 58.1 & 43.1 & 51.0 & 63.2 & $<0.0001$ \\
\hline High school or above (\%) & 3.9 & 6.7 & 4.5 & 3.4 & $<0.0001$ \\
\hline Married (\%) & 93.1 & 93.1 & 93.5 & 92.8 & 0.0008 \\
\hline Household income $\geq 35,000$ (yuan) (\%) & 37.8 & 40.9 & 38.7 & 37.0 & $<0.0001$ \\
\hline Current smokers (\%) & 28.0 & 26.7 & 27.6 & 28.5 & $<0.0001$ \\
\hline Current drinkers (\%) & 17.1 & 17.9 & 17.7 & 16.7 & 0.0011 \\
\hline Physical activities (MET-h/d) & $30.6 \pm 15.3$ & $26.4 \pm 16.3$ & $29.3 \pm 14.9$ & $31.6 \pm 15.1$ & $<0.0001$ \\
\hline Consuming meat daily (\%) & 15.2 & 26.9 & 16.1 & 13.8 & $<0.0001$ \\
\hline Consuming fruit daily (\%) & 6.7 & 16.7 & 7.8 & 5.5 & $<0.0001$ \\
\hline Consuming vegetable daily (\%) & 93.8 & 93.7 & 92.9 & 94.3 & $<0.0001$ \\
\hline $\mathrm{BMI}\left(\mathrm{kg} / \mathrm{m}^{2}\right)$ & $22.9 \pm 3.1$ & $23.2 \pm 3.3$ & $23.0 \pm 3.1$ & $22.8 \pm 3.1$ & $<0.0001$ \\
\hline WC (cm) & $76.5 \pm 9.1$ & $77.7 \pm 9.6$ & $76.7 \pm 9.1$ & $76.3 \pm 8.9$ & $<0.0001$ \\
\hline Sleep duration (hours) & $7.6 \pm 1.2$ & $8.0 \pm 1.3$ & $7.8 \pm 1.2$ & $7.5 \pm 1.1$ & $<0.0001$ \\
\hline Postmenopausal (\%) ${ }^{\mathrm{a}}$ & 53.7 & 53.8 & 53.7 & 53.7 & 0.86 \\
\hline
\end{tabular}

Data were adjusted for age and sex, as appropriate

MET metabolic equivalent tasks, BMI Body Mass Index, WC waist circumference

a Only among the females 
Table 2 Percentage of habitual daytime napping by different characteristics

\begin{tabular}{|c|c|c|c|c|c|}
\hline \multirow[t]{2}{*}{ Characteristics } & \multirow[t]{2}{*}{ Participants } & \multicolumn{2}{|c|}{ Habitual daytime napping } & \multirow[t]{2}{*}{$\mathrm{x}^{2}$} & \multirow[t]{2}{*}{$P$} \\
\hline & & $\mathrm{n}$ & Percentage $(95 \% \mathrm{Cl})$ & & \\
\hline Age range (years) & & & & 89.61 & $<0.0001$ \\
\hline $30-49$ & 23,099 & 1036 & $4.49(4.22-4.75)$ & & \\
\hline $50-59$ & 18,516 & 857 & $4.63(4.33-4.93)$ & & \\
\hline $60-79$ & 12,301 & 864 & $7.02(6.57-7.48)$ & & \\
\hline Sex & & & & 339.20 & $<0.0001$ \\
\hline Males & 22,573 & 1619 & $7.17(6.89-7.45)$ & & \\
\hline Females & 31,343 & 1138 & $3.63(3.46-3.80)$ & & \\
\hline
\end{tabular}

non-nappers, the adjusted HRs for risk of diabetes were 1.55 (95\% CI 1.35-1.77). The corresponding figures for males and females were 1.67 (95\% CI 1.38-2.01) and 1.43 (95\% CI 1.16-1.75), respectively. After additional adjustment for BMI and WC, the corresponding HRs for risk of incident diabetes among all of the participants were attenuated to be 1.41 (95\% CI 1.23-1.62). The corresponding figures for males and females were 1.47 (95\% CI 1.22-1.76) and 1.33 (95\% CI 1.08-1.63), respectively. After additional adjustment for self-reported snoring, habitual nappers had a 39\% higher risk of diabetes than non-nappers. Similar positive associations were found among males $(\mathrm{HR}=1.45,95 \% \mathrm{CI} 1.20-1.74)$ and females $(\mathrm{HR}=1.30,95 \%$ CI 1.05-1.59). (Table 3).

For participants who nap only in summer, after adjusting for socio-demographic status, cigarettes, alcohol, physical activity, dietary consumption, sleep duration, $\mathrm{BMI}$ and $\mathrm{WC}$, in comparison with non-nappers, the adjusted HRs for risk of incident diabetes were 1.06 (95\% CI 0.99-1.14). The corresponding figures for males and females were 1.09 (95\% CI 0.96-1.23) and 1.05 (95\% CI 0.95-1.15), respectively. After additional adjustment for self-reported snoring, similar non-significant associations were found among all participants $(\mathrm{HR}=1.05,95 \%$ CI $0.97-1.13)$, males ( $\mathrm{HR}=1.07,95 \% \mathrm{CI} 0.95-1.21)$, and females $(\mathrm{HR}=1.03,95 \% \mathrm{CI} 0.93-1.13)$ (Table 3$)$.

\section{Subgroup analyses}

Among non-obese participants, after adjusting for sociodemographic status, behavior lifestyle, BMI, WC, and self-reported snoring, habitual nappers had a $44 \%$ higher risk of incident diabetes than non-nappers $(95 \%$ CI 1.18 1.75). The corresponding figures for obese participants was 1.35 (95\% CI 1.11-1.63). No multiplicative interaction was found between habitual napping and obesity measures $(\mathrm{P}>0.05)$ (Table 4).

Table 3 Adjusted hazard ratios for diabetes in relation to daytime napping among participants of Zhejiang

\begin{tabular}{|c|c|c|c|c|c|c|}
\hline & N. of participants & $\begin{array}{l}\text { N. of incident } \\
\text { diabetes }\end{array}$ & $\begin{array}{l}\text { Model } 1 \\
\text { HR }(95 \% \mathrm{Cl})\end{array}$ & $\begin{array}{l}\text { Model } 2 \\
\text { HR }(95 \% \mathrm{Cl})\end{array}$ & $\begin{array}{l}\text { Model } 3 \\
\text { HR }(95 \% \mathrm{Cl})\end{array}$ & $\begin{array}{l}\text { Model } 4 \\
\text { HR }(95 \% \mathrm{CI})\end{array}$ \\
\hline \multicolumn{7}{|l|}{ Total } \\
\hline Non-napping & 33,511 & 1942 & 1 & 1 & 1 & 1 \\
\hline Napping only in summer & 17,648 & 1145 & $1.11(1.04-1.20)$ & $1.09(1.01-1.18)$ & $1.06(0.99-1.14)$ & $1.05(0.97-1.13)$ \\
\hline Habitual daytime napping & 2757 & 246 & $1.62(1.42-1.85)$ & $1.55(1.35-1.77)$ & $1.41(1.23-1.62)$ & $1.39(1.21-1.59)$ \\
\hline \multicolumn{7}{|l|}{ Males } \\
\hline Non-napping & 12,265 & 599 & 1 & 1 & 1 & 1 \\
\hline Napping only in summer & 8689 & 505 & $1.16(1.03-1.31)$ & $1.13(1.01-1.28)$ & $1.09(0.96-1.23)$ & $1.07(0.95-1.21)$ \\
\hline Habitual daytime napping & 1619 & 145 & $1.81(1.50-2.16)$ & $1.67(1.38-2.01)$ & $1.47(1.22-1.76)$ & $1.45(1.20-1.74)$ \\
\hline \multicolumn{7}{|l|}{ Females } \\
\hline Non-napping & 21,246 & 1343 & 1 & 1 & 1 & 1 \\
\hline Napping only in summer & 8959 & 640 & $1.09(0.99-1.20)$ & $1.08(0.98-1.18)$ & $1.05(0.95-1.15)$ & $1.03(0.93-1.13)$ \\
\hline Habitual daytime napping & 1138 & 101 & $1.45(1.17-1.76)$ & $1.43(1.16-1.75)$ & $1.33(1.08-1.63)$ & $1.30(1.05-1.59)$ \\
\hline
\end{tabular}

Model 1, adjusted for age and sex. Model 2, further adjusted for education level (no formal education, primary school, middle school, and high school or above), household income (<19,999 yuan, 20,000-34,999 yuan, and $\geq 35,000$ yuan), marital status, cigarettes consumption (never, occasional, former, and current regular), alcohol consumption (never, occasional, former, and current regular), meat, fresh fruits, and fresh vegetables consumption (daily and non-daily), physical activity (continuous) and sleep duration (continuous). Model 3, further adjusted for and BMI (continuous) and WC (continuous). Model 4, further adjusted for snoring (none, occasional, and habitual) 
Among postmenopausal females, after adjusting for socio-demographic status, behavior lifestyle, BMI, WC, and self-reported snoring, habitual daytime nappers had a $41 \%$ higher risk of diabetes than non-nappers (95\% CI 1.08-1.80). However, null statistically significant association was found among premenopausal females $(\mathrm{HR}=1.13,95 \%$ CI $0.78-1.59)$. (Table 5).

\section{Sensitivity analyses}

The associations of habitual daytime napping with diabetes did not changed appreciably after exclusion of the first 2 years of follow-up to reduce the effect of reverse causality, exclusion of those with diabetes family history to reduce the effect of family history. (Additional file 1: Table S1).

\section{Discussion}

In this large prospective study, the association of daytime napping with incident diabetes was explored. Habitual daytime napping was observed to be positively associated with risk of incident diabetes. However, such positive association was not found among premenopausal females.

Table 4 Adjusted hazard ratios for diabetes in relation to daytime napping among non-obese and obese participants

\begin{tabular}{|c|c|c|c|c|c|c|}
\hline & N. of participants & $\begin{array}{l}\text { N. of incident } \\
\text { diabetes }\end{array}$ & Model 1 & Model 2 & Model 3 & Model 4 \\
\hline & & & $\mathrm{HR}(95 \% \mathrm{Cl})$ & $\mathrm{HR}(95 \% \mathrm{Cl})$ & $\mathrm{HR}(95 \% \mathrm{Cl})$ & $\mathrm{HR}(95 \% \mathrm{Cl})$ \\
\hline \multicolumn{7}{|l|}{ Non-obese $\left(\mathrm{BMI}<25 \mathrm{~kg} / \mathrm{m}^{2}\right)$} \\
\hline Non-napping & 25,470 & 1027 & 1 & 1 & 1 & 1 \\
\hline Napping only in summer & 13,217 & 582 & $1.09(0.98-1.20)$ & $1.08(0.97-1.20)$ & $1.07(0.97-1.19)$ & $1.06(0.95-1.17)$ \\
\hline Habitual daytime napping & 2032 & 121 & $1.56(1.28-1.87)$ & $1.54(1.26-1.86)$ & $1.47(1.20-1.78)$ & $1.44(1.18-1.75)$ \\
\hline \multicolumn{7}{|l|}{ Obese $\left(\mathrm{BMI} \geq 25 \mathrm{~kg} / \mathrm{m}^{2}\right)$} \\
\hline Non-napping & 8041 & 915 & 1 & 1 & 1 & 1 \\
\hline Napping only in summer & 4431 & 563 & $1.08(0.97-1.20)$ & $1.06(0.95-1.18)$ & $1.06(0.95-1.18)$ & $1.04(0.93-1.16)$ \\
\hline Habitual daytime napping & 725 & 125 & $1.47(1.21-1.77)$ & $1.43(1.18-1.73)$ & $1.38(1.13-1.66)$ & $1.35(1.11-1.63)$ \\
\hline
\end{tabular}

Model 1, adjusted for age and sex. Model 2, further adjusted for education level (no formal education, primary school, middle school, and high school or above), household income (<19,999 yuan, 20,000-34,999 yuan, and $\geq 35,000$ yuan), marital status, cigarettes consumption (never, occasional, former, and current regular), alcohol consumption (never, occasional, former, and current regular), meat, fresh fruits, and fresh vegetables consumption (daily and non-daily), physical activity (continuous) and sleep duration (continuous). Model 3, further adjusted for and BMI (continuous) and WC (continuous). Model 4, further adjusted for snoring (none, occasional, and habitual)

Table 5 Adjusted hazard ratios for diabetes in relation to daytime napping among postmenopausal and premenopausal females

\begin{tabular}{|c|c|c|c|c|c|c|}
\hline & N. of participants & $\begin{array}{l}\text { N. of incident } \\
\text { diabetes }\end{array}$ & Model1 & Model2 & Model3 & Model4 \\
\hline & & & HR $(95 \% \mathrm{Cl})$ & $\mathrm{HR}(95 \% \mathrm{Cl})$ & $\mathrm{HR}(95 \% \mathrm{Cl})$ & HR $(95 \% \mathrm{Cl})$ \\
\hline \multicolumn{7}{|l|}{ Postmenopausal females } \\
\hline Non-napping & 11,047 & 849 & 1 & 1 & 1 & 1 \\
\hline Napping only in summer & 5192 & 443 & $1.12(0.99-1.25)$ & $1.10(0.98-1.24)$ & $1.07(0.95-1.20)$ & $1.04(0.93-1.17)$ \\
\hline Habitual daytime napping & 581 & 67 & $1.55(1.20-1.97)$ & $1.50(1.15-1.92)$ & $1.44(1.11-1.85)$ & $1.41(1.08-1.80)$ \\
\hline \multicolumn{7}{|l|}{ Premenopausal females } \\
\hline Non-napping & 10,199 & 494 & 1 & 1 & 1 & 1 \\
\hline Napping only in summer & 3767 & 197 & $1.03(0.87-1.21)$ & $1.03(0.87-1.21)$ & $1.02(0.86-1.21)$ & $1.01(0.85-1.20)$ \\
\hline Habitual daytime napping & 557 & 34 & $1.27(0.88-1.77)$ & $1.28(0.88-1.79)$ & $1.16(0.80-1.62)$ & $1.13(0.78-1.59)$ \\
\hline
\end{tabular}

Model 1, adjusted for age. Model 2, further adjusted for education level (no formal education, primary school, middle school, and high school or above), household income ( $<19,999$ yuan, 20,000-34,999 yuan, and $\geq 35,000$ yuan), marital status, cigarettes consumption (never, occasional, former, and current regular), alcohol consumption (never, occasional, former, and current regular), meat, fresh fruits, and fresh vegetables consumption (daily and non-daily), physical activity (continuous) and sleep duration (continuous). Model 3, further adjusted for and BMI (continuous) and WC (continuous). Model 4, further adjusted for snoring (none, occasional, and habitual) 


\section{Percentage of habitual daytime napping}

The percentage of habitual daytime napping in our study was $5.11 \%$, much lower than the average level of CKB in ten study regions $(20.3 \%)$. This difference reflected geographic diversity on distribution of daytime napping in China [10]. Consistent with the previous studies [10, 17], the percentage of habitual daytime napping increased with increasing age. Similar with the previous studies [7, $10,17]$, the percentage of habitual daytime napping was higher in males than in females in the present study.

\section{Association of daytime napping with diabetes}

One meta-analysis, including 304,885 individuals from four cross-sectional and six longitudinal studies, indicated that person with daytime napping had higher risk of diabetes in both cross-sectional and cohort studies than those without daytime napping [18], which was compatible with the present study. Another meta-analysis of 249,077 participants and 13,237 incident diabetes, comprising 7 prospective studies (one from the USA, two from China, and four from Europe), found that person with habitual daytime napping were $17 \%$ more likely to develop diabetes in comparison with those without daytime napping ( $R R=1.17,95 \% \mathrm{CI} 1.08-1.27)$. By region, while relative risks $(95 \% \mathrm{CI})$ of diabetes for the USA, and Europe were 1.21 (1.17-1.26) and 1.15 (1.03-1.30), respectively. No significant association of daytime napping with diabetes was found among Chinese adults $(\mathrm{RR}=1.23,95 \%$ CI $0.87-1.73)$ [19].

Contrary to one previous study performed in Guangzhou indicating that no significant association of daytime napping with prevalent diabetes was identified among Chinese males [17], the present study demonstrated that males with habitual daytime napping had a $45 \%$ higher risk for incident diabetes compared with the counterparts without napping after adjustment for socio-demographic status, behavioral lifestyle, BMI, WC, and self-reported snoring.

Previous studies reported that the effect of obesity on association of daytime napping with risk of diabetes was controversial. Hublin et al. found that the association of habitual nappers was associated with an increased risk for diabetes [12], but this association become non-significant after further adjustment for BMI, and concluded that napping is not an independent risk for diabetes. However, other studies, conducted in UK [11], USA [20], and China [21] showed that associations of habitual daytime napping with diabetes after adjustment for BMI were somewhat attenuated, but still statistically significant, consistent with our results. Health $A B C$ study showed that diabetic patients had higher odds of daytime napping compared to those without diabetes [22], and concluded that daytime napping might be a consequence of diabetes. In the current study, both self-reported physician-diagnosed diabetes and screen-detected diabetes at baseline were excluded from the final analysis, which ensured a temporal sequence between daytime napping and diabetes.

\section{Subgroup analysis}

To better observe the effect of obesity on the association of daytime napping with diabetes, analysis were conducted in both non-obese and obese population group separately. Notably, association between habitual daytime napping and risk of incident diabetes was significant not only in obese population group, but also in non-obese population group. This findings address the importance of reducing daytime napping among population with normal weight. Furthermore, because obesity has been widely accepted as an important risk for diabetes, our findings of significant association in non-obese population imply that besides obesity, there are other possible mechanisms explaining association of daytime napping with incident diabetes.

Menopause was associated with an adverse metabolic profile that increase the risk of diabetes [23]. However, few studies have observed the association of daytime napping with diabetes among premenopausal and postmenopausal women. One cross-sectional study including 6940 females aged 45 years or older from China indicated middle-aged postmenopausal women with more than 60 daytime napping per day had an $81 \%$ higher risk of diabetes in comparison with those without daytime napping. No significant differences were found among middleaged premenopausal women [24], which was in line with our findings.Future prospective studies are needed to explore the association of habitual daytime napping with diabetes in premenopausal women.

\section{Possible mechanisms}

There are several possible mechanisms explaining causal association of daytime napping with increased risk of diabetes. First, long daytime napping might disturb circadian rhythms [25], with increased night awakening and reduced nocturnal sleep duration [26], and subsequently, caused insulin resistance and diabetes [27, 28]. Additionally, daytime napping was recognized as a compensation for sleep loss or poor sleep quality due to increasing sleep fragmentation resulting from sleep disorders [29]. Sleep loss and poor sleep quality might contribute to the development of diabetes [30, 31]. Nevertheless, the association between daytime napping and nocturnal sleep was not completely identical. Previous studies indicated that duration of daytime napping appeared to be irrelevant to neither duration of nocturnal sleep nor its quality [20, 32]. Hence, association of daytime sleep with nocturnal 
sleep are needed to be explored in the future. Second, daytime napping was positively associated with inflammatory markers, including serum $\mathrm{C}$ reactive protein [33], which might enhance the development of diabetes [34, 35]. Third, daytime napping was an indicator of obstructive sleep apnea (OSA) [36]. OSA is characterized by the recurrent collapse of respiratory structures [37], which could increase sympathetic nervous activity and oxidative stress, and eventually cause insulin resistance [38]. Lastly, long daytime napping might decrease physical activity, resulting obesity and diabetes [39]. This assumption was also proven in our study that participants with habitual daytime napping had much less physical activity than non-nappers.

\section{Strengths and limitations}

The strength of this study include a large sample size, a prospective study design, and a relatively long follow-up duration. The exclusion of diagnosed and undiagnosed diabetes at baseline reduced the potential of reverse causality. The study also has several limitations. First, assessment of daytime napping was qualitative, lacking information on napping duration in questionnaire, restricts the ability to further explore association of short and long daytime napping duration with risk of incident diabetes. One previous study indicated that short daytime napping ( $<60 \mathrm{~min} /$ day) was not related to diabetes, and long napping was significantly associated with an increased risk of diabetes [40]. Second, although several established and potential risk factors for incident diabetes were adjusted for in different models, residual confounding by other unmeasured or unknown biological and social factors was still possible.

\section{Conclusions}

In conclusion, the current study presents that habitual daytime napping is positively related to diabetes in Chinese adults, except premenopausal females.

\section{Supplementary information}

The online version contains supplementary material available at https://doi. org/10.1186/s12986-021-00545-4.

Additional file 1: Table S1. Adjusted hazard ratios for diabetes in relation to daytime napping among specific participants.

\section{Abbreviations}

CKB: China Kadoorie Biobank; BMI: Body mass index; WC: Waist circumference; ICD: International classification of diseases; HRs: Hazard ratios.

\section{Acknowledgements}

The authors thank the all subjects for their participating in our study, all members of survey teams in the 10 regional centers for their efforts in baseline survey and follow-up survey. The authors also acknowledge support received from local Health Insurance Bureau for providing hospital admission data.

\section{Authors' contributions}

HW conceived and designed the study. ZC and LL, as the members of CKB steering Committee, designed the whole study. HW and MY analyzed the data and wrote the manuscript. LC, DS, YC, XZ, KX, CW, and SZ collected the data. YG supervised the implementation. BF provided critical comments on the manuscript and revised the manuscript. All the authors have read and approved the final submitted version.

\section{Funding}

The work was funded by Grants (2016YFC0900500, 2016YFC0900501, 2016YFC0900502, 2016YFC0900504) from National Key Research and Development Program of China, Grants from the Kadoorie Charitable Foundation in Hong Kong and Grants (202922/Z/16/Z, 104085/Z/14/Z, 088158/Z/09/Z) from Wellcome Trust in the UK. The funding sponsors have no role in design and conduct of the study; collection, analyses, and interpretation of the results.

\section{Availability of data and materials}

Details of how to access China Kadoorie Biobank data and details of the data release schedule are available from www.ckbiobank.org/site/ Data + Access.

\section{Ethics approval and consent to participate}

The CKB study was approved by the Ethical Review Committee of the Chinese Center for Disease Control and Prevention and the Oxford Tropical Research Ethics Committee, University of Oxford. All participants eligible for this study had completed a written informed consent form prior to the survey.

\section{Consent for publication}

Not applicable.

\section{Competing interests}

The authors declare that they have no competing interests.

\section{Author details \\ ${ }^{1}$ Department of NCDs Control and Prevention, Zhejiang Provincial Center for Diseases Control and Prevention, \#3399 Binsheng Road, Binjiang District, Hangzhou, Zhejiang Province, China. ${ }^{2}$ Department of NCDS Control and Prevention, Tongxiang City Center for Disease Control and Prevention, Tongxiang, China. ${ }^{3}$ Chinese Academy of Medical Sciences, Beijing, China. ${ }^{4}$ Medical Research Council Population Health Research Unit, Nuffield Depart- ment of Population Health, University of Oxford, Oxford, UK. ${ }^{5}$ Department of Epidemiology and Biostatistics, School of Public Health, Peking University, Beijing, China.}

Received: 23 October 2020 Accepted: 15 January 2021

Published online: 08 February 2021

\section{References}

1. Yang JJ, Yu D, Wen W, Saito E, Rahman S, Shu XO, Chen Y, Gupta PC, Gu D, Tsugane S, et al. Association of diabetes with all-cause and cause-specific mortality in Asia: a pooled analysis of more than 1 million participants. JAMA Netw Open. 2019:2:e192696.

2. Wang H, Ba Y, Cai RC, Xing Q. Association between diabetes mellitus and the risk for major cardiovascular outcomes and all-cause mortality in women compared with men: a meta-analysis of prospective cohort studies. BMJ Open. 2019:9:e024935.

3. The IDF Diabetes Atlas, 8th Edition [https://diabetesatlas.org/across-theglobe.html]

4. Ramachandran A, Ma RC, Snehalatha C. Diabetes in Asia. Lancet. 2010:375:408-18.

5. Wang L, Gao P, Zhang M, Huang Z, Zhang D, Deng Q, Li Y, Zhao Z, Qin X Jin $D$, et al. Prevalence and ethnic pattern of diabetes and prediabetes in China in 2013. JAMA. 2017:317:2515-23.

6. Bommer C, Heesemann E, Sagalova V, Manne-Goehler J, Atun R, Barnighausen T, Vollmer $\mathrm{S}$. The global economic burden of diabetes in adults aged 20-79 years: a cost-of-illness study. Lancet Diabetes Endocrinol. 2017;5:423-30.

7. Papandreou C, Diaz-Lopez A, Babio N, Martinez-Gonzalez MA, Bullo M, Corella D, Fito M, Romaguera D, Vioque J, Alonso-Gomez AM, et al. Long daytime napping is associated with increased adiposity and type 2 
diabetes in an elderly population with metabolic syndrome. J Clin Med. 2019;8:1053.

8. Lucassen E. To nap or not to nap: is the Wujiao a healthy habit? Sleep Med. 2013;14:929-30.

9. He J, Ouyang F, Qiu D, Duan Y, Luo D, Xiao S. Association of nap duration after lunch with prevalence of metabolic syndrome in a Chinese Government employee population. Int J Environ Res Public Health. 2020;17:4268.

10. Zheng B, Lin LL, Yu CQ, Lyu J, Guo Y, Bian Z, Tan YL, Pei P, Chen JS, Chen $Z M$, et al. Distributions and associations between duration of sleep, daytime naps and insomnia symptoms among Chinese adults. Zhonghua Liu Xing Bing Xue Za Zhi. 2017;38:452-6.

11. Leng Y, Cappuccio FP, Surtees PG, Luben R, Brayne C, Khaw KT. Daytime napping, sleep duration and increased 8-year risk of type 2 diabetes in a British population. Nutr Metab Cardiovasc Dis. 2016:26:996-1003.

12. Hublin C, Lehtovirta M, Partinen M, Koskenvuo M, Kaprio J. Napping and the risk of type 2 diabetes: a population-based prospective study. Sleep Med. 2016;17:144-8.

13. Chen Z, Lee L, Chen J, Collins R, Wu F, Guo Y, Linksted P, Peto R. Cohort profile: the Kadoorie Study of Chronic Disease in China (KSCDC). Int J Epidemiol. 2005:34:1243-9.

14. Chen Z, Chen J, Collins R, Guo Y, Peto R, Wu F, Li L. China Kadoorie Biobank collaborative g: China Kadoorie Biobank of 0.5 million people: survey methods, baseline characteristics and long-term follow-up. Int J Epidemiol. 2011:40:1652-66.

15. Li LM, Lv J, Guo Y, Collins R, Chen JS, Peto R, Wu F, Chen ZM. China Kadoorie Biobank Collaborative G: [The China Kadoorie Biobank: related methodology and baseline characteristics of the participants]. Zhonghua Liu Xing Bing Xue Za Zhi. 2012;33:249-55.

16. Kanazawa M, Yoshiike N, Osaka T, Numba Y, Zimmet P, Inoue S. Criteria and classification of obesity in Japan and Asia-Oceania. World Rev Nutr Diet. 2005:94:1-12.

17. Sun $K, L i F, Q i Y$, Lin $D$, Ren $M, X u M, L i F, L i Y$, Yan L. Sex difference in the association between habitual daytime napping and prevalence of diabetes: a population-based study. Endocrine. 2016;52:263-70.

18. Guo VY, Cao B, Wong CKH, Yu EYT. The association between daytime napping and risk of diabetes: a systematic review and meta-analysis of observational studies. Sleep Med. 2017;37:105-12.

19. Chen GC, Liu MM, Chen LH, Xu JY, Hidayat K, Li FR, Qin LQ. Daytime napping and risk of type 2 diabetes: a meta-analysis of prospective studies. Sleep Breath. 2018;22:815-24.

20. Xu Q, Song Y, Hollenbeck A, Blair A, Schatzkin A, Chen H. Day napping and short night sleeping are associated with higher risk of diabetes in older adults. Diabetes Care. 2010;33:78-83.

21. Fang $W, L i Z$, Wu L, Cao Z, Liang Y, Yang H, Wang Y, Wu T. Longer habitual afternoon napping is associated with a higher risk for impaired fasting plasma glucose and diabetes mellitus in older adults: results from the Dongfeng-Tongji cohort of retired workers. Sleep Med. 2013;14:950-4.

22. Goldman SE, Hall M, Boudreau R, Matthews KA, Cauley JA, Ancoli-Israel S, Stone KL, Rubin SM, Satterfield S, Simonsick EM, Newman AB. Association between nighttime sleep and napping in older adults. Sleep. 2008:31:733-40.

23. Slopien R, Wender-Ozegowska E, Rogowicz-Frontczak A, Meczekalski B, Zozulinska-Ziolkiewicz D, Jaremek JD, Cano A, Chedraui P, Goulis DG, Lopes $P$, et al. Menopause and diabetes: EMAS clinical guide. Maturitas. 2018;117:6-10.

24. Fang S, Zhou J. Association of daytime napping and diagnosed diabetes in middle-aged premenopausal, middle-aged postmenopausal, and older postmenopausal Chinese Women. Am J Health Promot. 2019;33:1107-14.
25. Feinberg I, Maloney T, March JD. Precise conservation of NREM period 1 (NREMP1) delta across naps and nocturnal sleep: implications for REM latency and NREM/REM alternation. Sleep. 1992;15:400-3.

26. Yoon IY, Kripke DF, Elliott JA, Youngstedt SD, Rex KM, Hauger RL. Agerelated changes of circadian rhythms and sleep-wake cycles. J Am Geriatr Soc. 2003;51:1085-91.

27. Cappuccio FP, D'Elia L, Strazzullo P, Miller MA. Quantity and quality of sleep and incidence of type 2 diabetes: a systematic review and metaanalysis. Diabetes Care. 2010;33:414-20.

28. Tasali E, Leproult R, Spiegel K. Reduced sleep duration or quality: relationships with insulin resistance and type 2 diabetes. Prog Cardiovasc Dis. 2009:51:381-91.

29. Grandner MA, Drummond SP. Who are the long sleepers? Towards an understanding of the mortality relationship. Sleep Med Rev. 2007;11:341-60.

30. Holliday EG, Magee CA, Kritharides L, Banks E, Attia J. Short sleep duration is associated with risk of future diabetes but not cardiovascular disease: a prospective study and meta-analysis. PLoS ONE. 2013;8:e82305.

31. Li YY, Hu JJ, Cheng MN, Yang QP, Wu F, Fu C, Shi Y. Relationship between both quality and duration of sleep and type 2 diabetes in middle-aged and elderly people in Shanghai. Zhonghua Liu Xing Bing Xue Za Zhi. 2020;41:1261-5.

32. Dautovich ND, McCrae CS, Rowe M. Subjective and objective napping and sleep in older adults: are evening naps "bad" for nighttime sleep? J Am Geriatr Soc. 2008:56:1681-6.

33. Leng Y, Ahmadi-Abhari S, Wainwright NW, Cappuccio FP, Surtees PG, Luben R, Brayne C, Khaw KT. Daytime napping, sleep duration and serum C reactive protein: a population-based cohort study. BMJ Open. 2014:4:e006071.

34. Dehghan A, Kardys I, de Maat MP, Uitterlinden AG, Sijbrands EJ, Bootsma AH, Stijnen T, Hofman A, Schram MT, Witteman JC. Genetic variation, C-reactive protein levels, and incidence of diabetes. Diabetes. 2007:56:872-8.

35. Cheng $L$, Zhuang $H$, Yang $S$, Jiang $H$, Wang $S$, Zhang J. Exposing the causal effect of c-reactive protein on the risk of type 2 diabetes mellitus: a mendelian randomization study. Front Genet. 2018;9:657.

36. Wen $Y$, Pi FH, Guo P, Dong WY, Xie YQ, Wang XY, Xia FF, Pang SJ, Wu $Y C$, Wang YY, Zhang QY. Sleep duration, daytime napping, markers of obstructive sleep apnea and stroke in a population of southern China. Sci Rep. 2016;6:34689.

37. Punjabi NM. The epidemiology of adult obstructive sleep apnea. Proc Am Thorac Soc. 2008;5:136-43.

38. Iiyori N, Alonso LC, Li J, Sanders MH, Garcia-Ocana A, O'Doherty RM, Polotsky VY, O'Donnell CP. Intermittent hypoxia causes insulin resistance in lean mice independent of autonomic activity. Am J Respir Crit Care Med. 2007:175:851-7.

39. Lucassen EA, Rother Kl, Cizza G. Interacting epidemics? Sleep curtailment, insulin resistance, and obesity. Ann N Y Acad Sci. 2012;1264:110-34.

40. Yamada T, Shojima N, Yamauchi T, Kadowaki T. J-curve relation between daytime nap duration and type 2 diabetes or metabolic syndrome: a dose-response meta-analysis. Sci Rep. 2016;6:38075.

\section{Publisher's Note}

Springer Nature remains neutral with regard to jurisdictional claims in published maps and institutional affiliations. 